Marcela Eunice Pinilla R.*, Alexandra Toro O.**, Diana Carolina Melo Vera***

\title{
Una muestra de la arquitectura moderna en Tunja: la obra del arquitecto Ernesto Muñoz Navarro. Una historia nunca contada ${ }^{1}$
}

\author{
A sample of modern Tunja architecture: the work of architect \\ Ernesto Muñoz Navarro. A story never told before \\ Cómo citar:
}

Pinilla Rodriguez, M., Toro Ospina, A, D., \& Melo Vera. (2019). Una muestra de la arquitectura moderna en Tunja:

la obra del arquitecto Ernesto Muñoz Navarro. Una historia nunca contada. Designia, 7(1), 117-137.

El presente artículo hace parte del proyecto de investigación "Reconocer nuestro patrimonio. Arquitectura moderna de Tunja. Obra de arquitecto Ernesto Muñoz Navarro" realizada con el grupo PAME, adscrito a la Facultad de arquitectura, Diseño y Urbanismo de la Universidad de Boyacá en Tunja.

* Profesional en Arquitectura de la Universidad Javeriana. Especialista en Gerencia de Empresas Constructoras de la EAN. Master of Business Administration con especialidad en Gerencia de proyectos de la Universidad del Mar, Chile. Docente y Directora del programa de arquitectura de la Universidad de Boyacá, Colombia, integrante del grupo de investigación PAME. E-mail: marpinilla@uniboyacá.edu.co ORCID: https://orcid.org/0000-0002-0138-6043

**Arquitecta de la Universidad de Los Andes, Master of Science en Conservación de patrimonio y desarrollo urbano de la Technische Universität Dresden Alemania, doctorado en curso en la Bauhaus

Universität Weimar, docente de la Universidad de Boyacá, integrante del grupo de investigación PAME. E-mail: atoro@uniboyaca.edu.co ORCID: https://orcid.org/0000-0001-6493-2665

*** Estudiante de pregrado en Arquitectura de la Universidad de Boyacá, integrante del Semillero Apqua, auxiliar de investigación del grupo PAME.

E-mail: dcmelo@uniboyaca.edu.co ORCID: https://orcid.org/0000-0002-6332-4622
Palabras clave:

arquitectura moderna,

arquitectura colombiana,

historia de la arquitectura

Key words:

Modern architecture, colombian architecture, history of architecture

Recibido: 17/10/2019

Aceptado: 26/06/2020 


\section{Resumen:}

La vida diaria de la ciudad de Tunja, Boyacá, Colombia es testigo desde hace más de medio siglo de la arquitectura moderna; sin embargo, este estilo arquitectónico ha sido objeto de muy pocos estudios, tal vez debido a la preminente importancia que tiene para el habitante tunjano y para la academia, la imagen e identidad de Tunja como ciudad colonial, que, por tanto, prima sobre la imagen moderna de ciudad y sus manifestaciones arquitectónicas.

Este artículo recopila conclusiones preliminares y el avance de la investigación realizada sobre la vida y obra del Arquitecto e Ingeniero Ernesto Muñoz Navarro, pionero de la aplicación de los conceptos del modernismo en Tunja.

Los datos obtenidos nos acercan a la figura de Ernesto Muñoz Navarro y lo ubican en un momento clave del desarrollo del país en el cual la arquitectura moderna fue protagonista. Adicionalmente, el presente artículo hace una reflexión sobre la falta de reconocimiento del valor patrimonial de la arquitectura moderna, que es justamente lo que ha llevado a que la obra de Ernesto Muñoz Navarro sea totalmente desconocida.

Para iniciar el trabajo, se consideró fundamental realizar la investigación sobre la vida de este personaje, como aspecto fundamental para poder entender y apreciar su obra. Posteriormente, se indagó sobre el momento histórico en el cual el profesional del arte de la construcción Ernesto Muñoz Navarro construyó en Tunja edificaciones que marcaron el desarrollo de la ciudad. Un resumen de estas dos etapas del trabajo de investigación se consigna en este artículo, el cual brinda una reflexión sobre el valor que tiene la arquitectura moderna en la ciudad de Tunja y la necesidad de rescatar dicho valor desde la apropiación ciudadana y la investigación académica. 


\section{Abstract:}

City's daily urban life of Tunja, Boyacá, Colombia has been witness of the modern architecture for more than half a century; however, this architecture style has been object of few studies, due to the preeminent character historical of the city. Tunja citizens have been studying the colonial architecture, more than the modern architecture of the city. Therefore, the colonial identity of the city is more important than modern image of the city and them modern architecture manifestations, for academics and people.

This document collects preliminary conclusions and the research advances about the life and the work of the architect and engineer Ernesto Muñoz Navarro, who was a pioneer of the application of the concepts of the modernism, in Tunja, Boyacá.

The data obtained approach us to the figure of Ernesto Muñoz Navarro and let us situates him in a main time of the Colombia development in which the architecture was a protagonist. Additionally, this article gives a careful thought about the lack of recognition of the heritage value of the modern architecture whereby the Ernesto Muñoz Navarro work is completely unknown.

Firstly, it was fundamental began the research about the character life, like key part of understanding and appreciating of his work. Subsequently, we explored about the historic time in which this professional of the construction art built important buildings that have marked the urbanistic development of Tunja, Boyacá. A summary of these two stages research process is included in the present article, which gives a reflection about the value of modern architecture in the city and the need to recover the modernism architecture value from the citizen appropriation and the academic research. 


\section{INTRODUCCIÓN}

El presente artículo corresponde a la ponencia realizada para el $V$ Seminario Internacional Gestión del Patrimonio Cultural realizado en octubre de 2018, sobre el avance de la investigación acerca de la obra del Arquitecto e Ingeniero Ernesto Muñoz Navarro, en la ciudad de Tunja, durante las décadas de 1950 y 1960. La investigación está siendo realizada por docentes del grupo de investigación PAME de la Facultad de Arquitectura, Diseño y Urbanismo de la Universidad de Boyacá, con el apoyo de los estudiantes miembros del semillero de investigación $A P Q U A$, que están interesados en el patrimonio construido.

El proyecto de investigación se origina a raíz de la donación, por parte de la esposa de Ernesto Muñoz Navarro, del acervo documental de este profesional a la Universidad de Boyacá, después del fallecimiento de este ilustre personaje. La investigación inició en abril de 2017, coincidiendo con la creación del semillero de investigación APQUA, por lo cual se articularon el grupo de investigación PAME y este semillero, para iniciar el presente ejercicio académico.

Puesto que los planos fueron recibidos sin clasificar, la primera tarea consistió en ordenarlos. Algunos documentos correspondían a edificaciones diseñadas por él y en otros él figuraba como interventor que no diseñó ni construyó. Cabe resaltar, que de las obras más importantes en Tunja realizadas por Ernesto Muñoz, había un número limitado de planos con la característica de que estaban incompletos. Por esta razón, los estudiantes del semillero realizaron planos de levantamiento de algunas edificaciones, buscando reconstruir el acervo técnico documental base del proceso investigativo.

En ese orden de ideas, el objetivo del presente artículo es resumir los avances del proyecto ubicando la obra del Arquitecto e Ingeniero Ernesto Muñoz Navarro en un momento histórico del desarrollo urbanístico del país, en el cual la arquitectura moderna fue la principal protagonista. Lamentablemente, los valores de la arquitectura moderna no han sido reconocidos, por lo cual dicha importancia histórica ha pasado desapercibida, lo que está llevando a la invisibilización y desaparición de magníficas obras del modernismo que resumen la historia de Colombia de los últimos sesenta años. 


\section{PASOS INICIALES DE LA INVESTIGACIÓN}

La primera etapa de búsqueda de información comenzó por indagar sobre la vida de Ernesto Muñoz Navarro para comprender su forma de ser, en relación con las obras que se analizarían a futuro. Así, se realizaron entrevistas a los familiares, amigos y trabajadores del personaje. Gracias a los relatos de estas personas, se logró consolidar una completa biografía y, también, se consiguió identificar sus obras más importantes realizadas en Tunja, a pesar de no tener planos originales completos.

Seguidamente, se desarrolló un proceso de clasificación documental de los planos donados, lo cual arrojó la conclusión de que no todos los planos donados, eran obras de su autoría, pues otros eran de proyectos en los que participó indirectamente, sin diseñar ni construir y otros eran documentos de interventoría profesional.

Cabe resaltar, que en total se encontraron tres planos arquitectónicos con sus diseños más importantes desarrollados en la ciudad de Tunja, que no estaban completos. Por ende, desde el semillero de investigación APQUA, se desarrolló un proceso de elaboración de planos de levantamiento de aquellas edificaciones de las cuales no se disponía de plano original.

Después de organizar el acervo técnico documental, se desarrolló una reflexión sobre la importancia de las obras de arquitectura moderna y el papel que han jugado en el desarrollo y en la imagen de las ciudades colombianas, a partir del caso específico de la relación entre el modernismo desarrollado por nuestro personaje en estudio y el impacto que sus obras generaron en la ciudad de Tunja. En las siguientes líneas extenderemos esta reflexión.

\section{CONTEXTO HISTÓRICO. LA ARQUITECTURA MODERNA EN COLOMBIA EN LOS AÑOS 50}

Alberto Saldarriaga Roa, en su artículo "Arquitectura colombiana en el siglo XX: edificaciones en busca de ciudad" (1999), relata la evolución de la arquitectura moderna en Colombia: en un primer periodo, referenciado temporalmente en el período entre 1900 \& 1930, había una fuerte influencia academicista; las obras fueron desarrolladas por arquitectos europeos llegados al país por su propia voluntad o por encargos especiales, como Gastón Lelarge, Pietro Cantini, Robert M. Farrington, entre otros. 
Un segundo período, a partir de la década de 1930, es motivado por arquitectos extranjeros como Vicente Nasi, Leopoldo Rother y Bruno Violi; y se concreta finalmente con profesionales ingenieros y arquitectos colombianos formados a partir de las enseñanzas e influencias de los arquitectos extranjeros (1999, pág. 114).

La manera como los arquitectos colombianos aportaron al segundo período es aclarada por Eduardo Samper en su libro "Arquitectura moderna en Colombia: época de oro" (2000), que corresponde a un período de 20 años durante los cuales, algunos arquitectos, en su mayoría de la Universidad Nacional de Colombia, Sede Bogotá, promulgaron los conceptos de la arquitectura moderna en varias regiones del país.

A este grupo de profesionales del diseño urbanístico, perteneció el madrileño Ernesto Muñoz Navarro. Como anota Alberto Saldarriaga, gracias a ellos la arquitectura moderna fue entendida en el país como una muestra de progreso y renovación ciudadana e infra-estructural; así, los profesionales formados en las escuelas de arquitectura colombiana de este período, trabajaron para el sector público y para sector el privado, construyendo edificaciones con una nueva estética y nuevos materiales (Saldarriaga Roa, La arquitectura en Colombia en varios tiempos, 2017).

Es de aclarar que el contexto político y económico de Colombia en los años 50, facilitó el desarrollo de la arquitectura moderna. Sin embargo, las condiciones culturales y técnicas del país, hacen que esta dinámica se desarrolle en etapas. La arquitectura moderna llegó a Colombia en los años 50 del siglo XX y sus proyectos se extendieron hasta la década de los 60. Abarcó todo tipo de edificaciones, desde edificios públicos hasta residencias privadas, cuyos diseños retomaban las pautas determinadas por el movimiento moderno en Europa, que se venía dando desde la década de los 20 .

Los arquitectos colombianos retomaron características de esta arquitectura europea en sus obras, reconocieron las nociones esenciales de la arquitectura moderna representadas en simplificación de las formas, rechazo a los lenguajes clásicos, ausencia de ornamento, utilización de nuevos materiales y tecnologías, y las reinterpretaron, dando como resultado una arquitectura propia con particularidades tan originales como las de las obras que la inspiraron, logrando así una adecuada relación entre lo universal y lo local (Henao \& Llanos, 2008) (añadir pág.) 


\section{EL ARQUITECTO REPRESENTANTE DEL MODERNISMO ARQUITECTÓNICO}

El protagonista de la arquitectura moderna es el arquitecto, debido a su activa participación en el concepto estético de las edificaciones; en Colombia el profesional en el arte de la construcción inició trabajando de manera individual, pero también asociativamente, creando firmas de arquitectos e ingenieros, que manejaban la obra de manera integral, desde el diseño hasta su construcción. Varias firmas fueron muy famosas por el adecuado ejercicio de su profesión. Como afirma García de Moncada (2007),

\section{Llegar a ser moderno no es una decisión voluntaria, es casi una obligación en un mundo en el que las sociedades más poderosas son aquellas más modernas. En éstas la industrialización tiene origen y encuentra expansión en la transformación que se llevó a cabo en los ámbitos político, social, económico y cultural. (p.19).}

Ahora bien, por más que las obras arquitectónicas generaron grandes impactos en la composición de las ciudades, hoy están en el olvido al igual que los aportes que estos ejercicios profesionales dejaron al modernismo del país. De manera que, es fundamental sacar a la luz las obras constructivas y dar a conocer las características de estos desarrollos artísticos reflejados a través del espacio. (Henao \& Llanos, 2008)

En este punto de la reflexión, es preciso mencionar a la arquitecta Silvia Arango en su artículo: "Arquitectura colombiana de los años 30 y 40: la modernidad como ruptura (1997)". En la Colombia de los años 30, un arquitecto joven estaba influenciado por libros y revistas extranjeras inundadas de poesía y arquitectura moderna. En su entorno, las ciudades estaban aletargadas, con viejos edificios y pocos habitantes. La mayoría de ciudades, crecían y cambiaban lentamente sin dejar de modificar su apariencia y costumbres de la colonia, en profundo contraste con las grandes ciudades europeas, que ya atisbaban revoluciones en el diseño arquitectónico.

Por ese motivo, el limitado gremio de arquitectos colombianos de esta época, quiso "ponerse al día" destruyendo las usanzas tradicionales del quehacer arquitectónico, con miras a un futuro mejor, directamente relacionado con la arquitectura 
moderna de las revistas europeas. Fue así como inventaron una modernidad propia, a la que se unieron los arquitectos extranjeros que trabajaron en el país. El resultado fue una arquitectura moderna original, de un valor e influencia importante, que aún no ha sido reconocido:

\begin{abstract}
...La arquitectura moderna brevemente ilustrada con los ejemplos anteriores no puede interpretarse con los filtros estilísticos, tipológicos o constructivos europeos o norteamericanos. Es distinta. Es original. Tampoco surge espontáneamente de un movimiento social colectivo. Fue inducida por un grupo de profesionales de la arquitectura, identificables con nombre propio. Y significó una ruptura auténtica y radical con el pasado arquitectónico: después de su irrupción en nuestras ciudades, no hubo ya posibilidad de volver atrás. (Arango, 1997)
\end{abstract}

\title{
TUNJA EN LA DÉCADA DE LOS 50
}

Tunja siempre ha sido reconocida y valorada como una ciudad ejemplo de la arquitectura de la colonia. Sin embargo, a partir de la segunda década del siglo XX comienzan a darse modificaciones en su arquitectura; a partir de la celebración del primer centenario de la independencia, las obras que intervinieron el espacio público, tales como el Bosque de la República y el Parque de los Mártires, comienzan a caracterizar su imagen. Pero lo que realmente dio un vuelco a la imagen de ciudad colonial reconocida en Tunja, fue la construcción, en la década de los 50, de edificaciones modernas que modificaron su lenguaje arquitectónico, su configuración urbanística y su perfil homogéneo característico.

Gran parte de este cambio se debe al gobierno del tunjano, ingeniero y General Gustavo Rojas Pinilla, presidente de la República entre 1953 y 1957, cuyo mandato se caracterizó por la realización de grandes obras de infraestructura y por su interés en modernizar el país con sus planes de desarrollo elaborados por expertos en economía y educación, a fin de que Colombia estuviera a la par con otros países Latinoamericanos (García de Moncada, 2007, pág. 20).

Durante el gobierno del General Rojas Pinilla, se construyeron edificaciones modernas para representar el poder del Estado y sus transformaciones económicas. El gobierno de Rojas Pinilla ha sido el que mayor número de obras públicas en el Siglo XX ha construido. (García de Moncada, 2007, pág. 20).

Los ideales gubernamentales de modernizar al país durante este período, se unieron a las motivaciones estéticas de la nueva generación de arquitectos e 
ingenieros, quienes pudieron expresar sus conocimientos y avances en el diseño y la construcción. (García de Moncada, 2007, pág. 20). Esta idea la ratifica así Edmundo Quevedo Forero (1999):

\begin{abstract}
“La relación entre lo duradero con los sentimientos se relaciona en las frases del Ministro de Obras Públicas, Contralmirante Rubén Piedrahita: “...edificar algo estable, de calidad adecuada para resistir el óxido del tiempo y el corrosivo de la incomprensión, exige esfuerzo, dedicación y cariño y con solo nuestro soplo creamos formas aparentemente estables y permanentes en el vidrio. Fresco aún en el fruto de tan pequeño esfuerzo, el mismo soplo nuestro destruye esas formas y las reduce a polvo. En cambio, cuanto sudor de nuestra frente, cuanta fatiga de nuestro brazo, cuanta perseverancia de nuestro ánimo requerimos para forjar el acero, más la forma que nuestra sostenida vigilia imprime en el metal es capaz de resistir el tiempo, de desafiar la inclemencia de los elementos y de soportar la acción del hombre". (García de Moncada, 2007, pág 20)
\end{abstract}

En efecto, Rojas Pinilla fue el presidente que más obras ha realizado en el departamento de Boyacá (Redacción El Tiempo, 2000). Entre ellas se comprende el trazado de la carretera Belén - Socha - San Salvador, la instalación y puesta en funcionamiento de Cementos Boyacá, La Radiodifusora La Independencia, Termo Tunja, tres unidades en Termo Paipa, ampliación y pavimentación de la carretera Tunja-Barbosa, el acueducto de Teatinos para Tunja, las obras de acueductos de Sogamoso y Belencito, el Lago Sochagota de Paipa, la Industria Militar en Sogamoso, la ampliación y pavimentación de la Carretera Central del Norte entre Belencito y Paz del Río, el aeropuerto de Tunja, la cárcel de Tunja, el Palacio Municipal de Tunja y el Palacio de Justicia de Tunja (Redacción El Tiempo, 2000), siendo estas últimas protagonistas del cambio en la imagen de la capital Boyacense.

Ese cambio en la imagen y en la percepción del espacio tunjano generó diversas reacciones entre la ciudadanía de aquel entonces. Principalmente, las edificaciones sobre la Plaza de Bolívar, al modificar su perfil, generaron opiniones negativas de parte de quienes respetaban y valoraban lo tradicional. A partir de estas obras, se continuaron construyendo edificaciones modernas donde antes hubo casas coloniales (Hidalgo p. , 2014) 
Una muestra de la reacción que generó la arquitectura moderna la expresa Juan Medina Roa en su libro Tunja desde 1900 (Medina Roa, 2016, págs. 60-61), en el cual anota, en relación con los edificios modernos del costado sur de la Plaza de Bolívar: “El costado sur de la Plaza de Bolívar fue el más afectado por la destrucción. ... El impacto en el entorno fue indudable.... La mentalidad arrasadora, so pretexto de "modernizar", impactó fuertemente en Tunja." $Y$ añade: "Por los años cincuenta se desata una ola de construcciones que desconocieron las preexistencias de la época colonial. La ciudad fue tomando un aspecto de improvisación y caos estético."

Más adelante escribe: "El edificio de la alcaldía pareció generar una reacción en cadena. Se demolieron todas las antiguas casas del sur de la plaza, para construir el Edificio de la "Beneficencia" conocido como el Edificio de la Lotería y el edificio sede de Telecom, ... (pág. 65). Lo escrito por Juan Medina Roa muestra claramente la visión de quienes se encontraban aferrados a la imagen colonial de la ciudad y consideraban, y aun consideran que esta no debió cambiar y que debió haberse mantenido siempre el centro histórico homogéneo en fachadas altura y volumetría.

\section{EL DESCONOCIMIENTO DE LA ARQUITECTURA MODERNA EN TUNJA}

La arquitectura moderna en Boyacá, y específicamente en Tunja, ha sido muy poco estudiada. La publicación del Ministerio de Cultura: "Ciudad y arquitectura moderna en Colombia 1950-1970: Presencia y vigencia del patrimonio moderno" (2008), hace una selección de obras representativas de la modernidad en Colombia en las ciudades colombianas donde se encuentran ejemplos valiosos de modernidad arquitectónica, sin hacer ninguna mención de Tunja.

Son contadas las publicaciones que mencionan ejemplos de arquitectura moderna en Boyacá, y el cubrimiento bibliográfico que se realiza sobre la arquitectura moderna tunjana, es casi inexistente. Citando a Hidalgo (2015), él menciona en su artículo tres elementos modernos del patrimonio urbano difuso en Tunja, las instalaciones del aeropuerto Gustavo Rojas Pinilla, la Escuela Normal de Varones y los Silos del Instituto Nacional de Abastecimiento (INA).

De igual manera, Mendoza (2008) en su artículo: “La pérdida de la tradición moderna en la arquitectura colombiana: Bogotá y sus alrededores, y departamentos de Boyacá, Cundinamarca, Huila y Tolima", realiza un selección de obras de arquitectura moderna significativa en Bogotá, Boyacá, Cundinamarca, Huila y Tolima, correspondientes al periodo entre los años treinta y finales de los sesenta del siglo XX, analizando los cambios de que han sido objeto, como demoliciones y 
modificaciones que han alterado sus características originales (pág. 185). En Tunja menciona, dentro de las obras cuya ejecución es diferente a la diseñada, el Edificio Telecom, proyecto de Sokoloff y Camargo del año 1954 (pág. 189) y dentro de las obras que llama "desfiguradas" está la Escuela Normal de Tunja, del arquitecto Pablo de la Cruz, construida en 1935 (Mendoza Laverde, 2008, pág. 190).

En particular, el reconocimiento de la arquitectura moderna tunjana dentro de la reflexión académica es muy reducido y, específicamente, no incluye dentro de sus análisis, la obra del Arquitecto e Ingeniero Ernesto Muñoz Navarro de manera directa y rigurosa.

Del análisis académico sobre los diseños arquitectónicos de Ernesto Muñoz desarrollados a lo largo de su vida profesional en la ciudad de Tunja, se encuentran reseñados en algunos libros sobre la historia del siglo XX en Tunja, a manera de referencia indirecta.

Por ejemplo, en el libro: "Tunja desde 1900" de Medina Roa Juan, encontramos que respecto al edificio del Banco de la República, este fue construido por el arquitecto Ernesto Muñoz, quien siguió los lineamientos institucionales (Medina Roa, 2016, pág. 84).

Adicionalmente, también se encuentra el libro "Tunja: transformación urbana a partir de la vivienda obrera (1940 - 1957)" de Hidalgo (2014), donde muestra una fotografía del aviso publicitario del edificio de Banco del Comercio, con el sello de la firma "arquitectos y constructores Caicedo \& Muñoz Ltda". El aviso, publicado en la revista Boyacá (1956, Agosto No. 9), hace referencia a los inquilinos del edificio ubicados en el cuarto piso, donde también se hallaban las oficinas del Arquitecto Ernesto Muñoz Navarro. (Hidalgo A. , 2014, pág. 109)

Un avance en la investigación del patrimonio moderno en Tunja lo inició la Universidad Santo Tomás seccional Tunja, con el proyecto DOCOMOMO Centro oriente Colombia: "Avance de la investigación sobre arquitectura moderna en Boyacá", coordinado por la arquitecta Martha Cecilia López (2009). 
El estudio afirma que, si bien se evidencia un latente arraigo de la población Tunjana a la tradición, muchas edificaciones de la colonia declaradas "Bien de Interés Cultural para la Nación" se encuentran en mal estado de conservación, pues los ciudadanos no valoran la tradición arquitectónica; de igual forma, los ejemplos de arquitectura moderna son criticados pues representan la destrucción del "verdadero patrimonio" para construir un "supuesto progreso". Es así, como muestras fundamentales del patrimonio arquitectónico moderno ya han sido destruidas o transformadas sustancialmente.

Una de las razones para el desconocimiento del valor de la arquitectura moderna en Tunja se observa porque esta ciudad está caracterizada por su arquitectura histórica considerando que el Centro Histórico de Tunja fue declarado Monumento Nacional mediante la Ley 163 del 30 de diciembre de 1959, la cual delimitó el sector de las calles, plazas, plazoletas, inmuebles, casas y construcciones históricas, tejidos, muebles, etcétera, comprendidos dentro del perímetro que tenía la población durante los Siglos XVI, XVII y XVIII.

Acogiendo el concepto de Caballero y Fonseca en su libro: "Aproximación operativa al inventario - valoración del patrimonio construido" (2001, pág. 6), en 1959 y 1963 debía haber en Colombia más poblaciones con más méritos para recibir la clasificación de Monumento Nacional, lo que indica que esta declaratoria no obedeció a una valoración de todos los centros de la época de la colonia, y además se dio por hecho que lo arquitectónico y lo urbano de esta época era lo que tenía valor. Esto se aprecia en la lentitud con que han sido considerados patrimonio otros urbanismos y otras arquitecturas en Colombia, a lo largo del tiempo. 


\section{EL ARQUITECTO E INGENIERO ERNESTO MUÑOZ NAVARRO}

La biografía del ilustre profesional colombiano Ernesto Muñoz Navarro se elaboró gracias a las entrevistas realizadas a Marcela Muñoz, su hija; a Luis Arévalo, su dibujante por muchos años; al arquitecto Edilberto Molina, vecino de su casa sobre el Parque Santander, en la ciudad de Tunja; al arquitecto César Castellanos, pupilo del arquitecto Muñoz y al arquitecto Manuel Mejía, con sus aportes sobre la Tunja de la época de Muñoz Navarro.

Ernesto Muñoz Navarro nació en Madrid, Cundinamarca, en 1916. Fue uno de los primeros Arquitectos e Ingenieros egresados de la Universidad Nacional de Colombia en el año de 1941, en un momento en que la arquitectura moderna estaba en su apogeo en Colombia, influenciada por importantes profesionales extranjeros que llegaron al país a revolucionar el concepto de diseño y construcción arquitectónica.

Su abuelo hizo parte del equipo de trabajo del Puente Navarro sobre el Río Magdalena, en Honda, Tolima, lo que le permitió viajar con su familia a Bélgica, donde Ernesto Muñoz Navarro realizó sus estudios de primaria. A raíz de la Segunda Guerra Mundial, la familia regresó a Colombia, y Ernesto Muñoz Navarro y su hermano, terminaron sus estudios en Bogotá en el Instituto de la Salle.

Terminados sus estudios de bachillerato, viajó nuevamente a Bélgica, donde inició la carrera de arquitectura, pero a los pocos meses se trasladó a Chile a continuar sus estudios. Debido a la enfermedad de su padre, tuvo que regresar a Colombia, donde retomó su carrera en la Universidad Nacional y obtuvo el título de Arquitecto e Ingeniero el 21 de noviembre de 1941.

Poco tiempo después de graduarse, renovó el segundo sector de las instalaciones de Malterías de Santa Rosa de Viterbo, trabajo que le permitió conocer Boyacá y encantarse con este lugar, por lo que decidió establecerse en el departamento y casarse en 1951, con Beatriz Azuero.

Beatriz Azuero de Muñoz, fue una importante dama boyacense; ocupó varios cargos públicos en la ciudad de Tunja, llegando a ser Concejala de la ciudad, Sub-secretaria de Educación, Secretaria de Educación, Alcaldesa de Tunja (1965-1969), Delegada del Ministerio de Educación, Directora del FER en Boyacá, fundadora de la Cruz Roja y miembro del Consejo Superior de Policía. Asimismo, fue una gran líder cívica que creó y puso en marcha el Cuerpo de Bomberos Voluntarios de la ciudad. 
Ya radicado en Tunja, Ernesto Muñoz Navarro creó la firma constructora y de diseño Caice do y Muñoz L.T.D.A. Fue miembro fundador de la Asociación Colombiana de Ingenieros y Arquitectos, socio fundador del Rotary Club Internacional de Boyacá, gestor de la primera y única convención del Rotary Internacional en 1953, miembro de la Sociedad Boyacense de Ingenieros y Arquitectos y profesor de dibujo en la Universidad Pedagógica y Tecnológica de Colombia (UPTC).

Su primera oficina se localizó en el séptimo piso del edificio de la Beneficencia de Boyacá, en la ciudad de Tunja, proyecto para el cual realizó la interventoría de la obra; posteriormente, trasladó su oficina al tercer piso del edificio del Banco del Comercio, el cual él mismo diseñó y lideró en su etapa constructiva en el año de 1957. Dado lo novedoso de sus diseños para su época y por su personalidad, Ernesto Muñoz Navarro fue conocido como el Loco Muñoz. Un apunte interesante, es que a personas de la época se les pregunta sobre Ernesto Muñoz Navarro y no saben de quién se trata, pero al mencionarles al Loco Muñoz, caen en cuenta de quién se está hablando.

En la última etapa de su vida, Muñoz Navarro fue diagnosticado con diabetes. Aunque supo controlar esta enfermedad durante muchos años, finalmente el mal fue muy fuerte y quedó ciego en 1993. Su esposa renunció al trabajo, pues la diabetes de Muñoz empeoró gradualmente, hasta que falleció en 1994.

Hoy en día, la obra de El Loco Muñoz sigue en pie en varias ciudades y municipios colombianos por los que el arquitecto pasó a lo largo de su ejercicio profesional; las construcciones no han sido reconocidas como obras de significativa importancia para el movimiento moderno en la arquitectura colombiana y lamentablemente, algunas han sido modificadas en el tiempo debido a cambios de uso. 
De acuerdo a la información que fue obtenida de la revisión de los planos donados a la Universidad de Boyacá por la viuda del Ernesto Muñoz Navarro y las entrevistas enunciadas anteriormente, algunas de las obras más importantes construidas por el arquitecto Ernesto Muñoz Navarro en Boyacá son las siguientes:

- Cárcel de Santa Rosa de Viterbo

- Hospital Central de Puerto Boyacá

- Ampliación de la Siderúrgica de Paz de Río

- Ampliación de la Planta Eléctrica de Paipa

- Diseño de un conjunto de vivienda en Sogamoso.

- Diseño y construcción del Parque Tibaná.

Además, nuestro personaje, trabajó para la Arquidiócesis de Tunja, organización para la cual adelantó las siguientes obras:

- Diseño y construcción de la Iglesia de Otanche, (un proyecto diferente en su estilo por tener forma curva).

- Proyecto del Seminario Mayor.

- Reconstruyó la Capilla de Turmequé y diseñó el Parque Turmequé.

Y en la ciudad capital del departamento de Boyacá, adelantó las siguientes obras:

- Casa sobre el Parque Santander, sede inicial de la Universidad de Boyacá.

- Casa actual sede de Profamilia, la que por muchos años fue ocupada por los gerentes de la Industria Embotelladora de gaseosas de Boyacá.

- Dos casas en el barrio La María, una de ellas para Víctor Castro.

- Auditorio José Mosser.

- Casa de vivienda propia sobre el Parque Santander.

- Edificio Hermanos Fonseca.

- Una manzana construida con vivienda en la cercanía del barrio Maldonado.

- Edificio del Banco del Comercio, hoy Secretaría de Educación. 


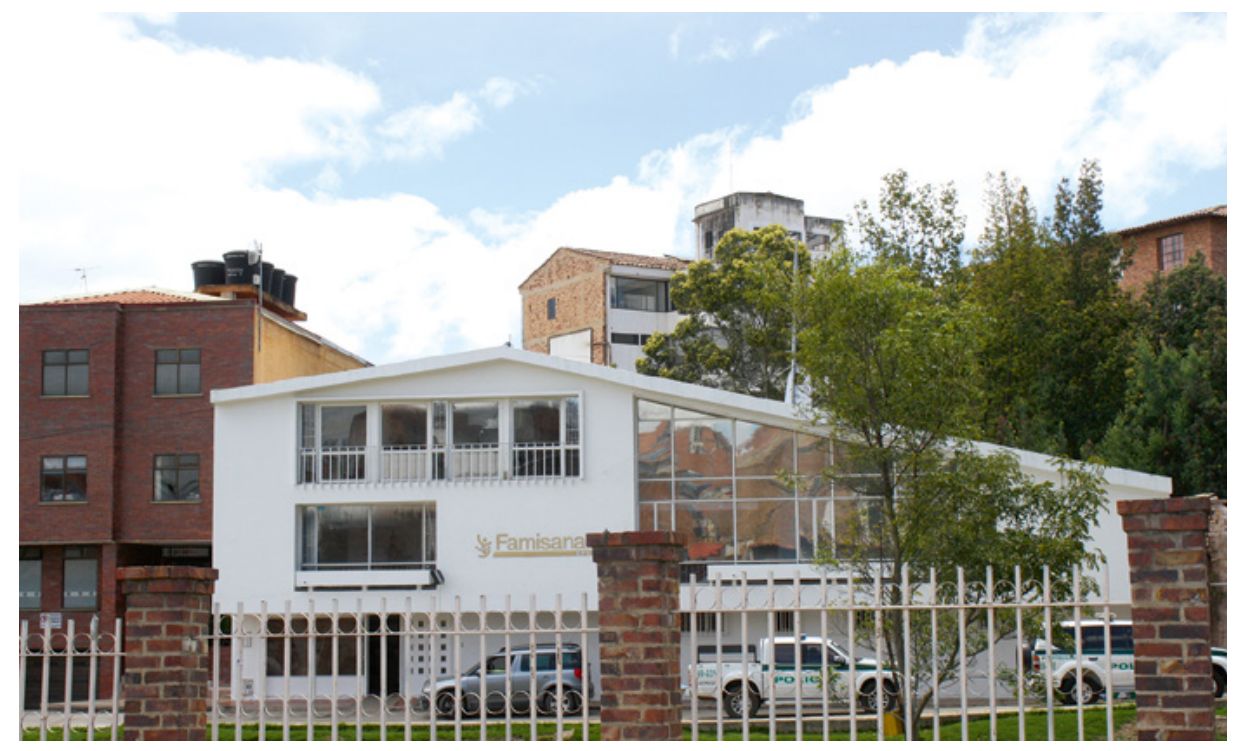

Figura 1. Casa del Ing. Arq. Ernesto Muñoz Navarro. Fuente: Marcela Pinilla.

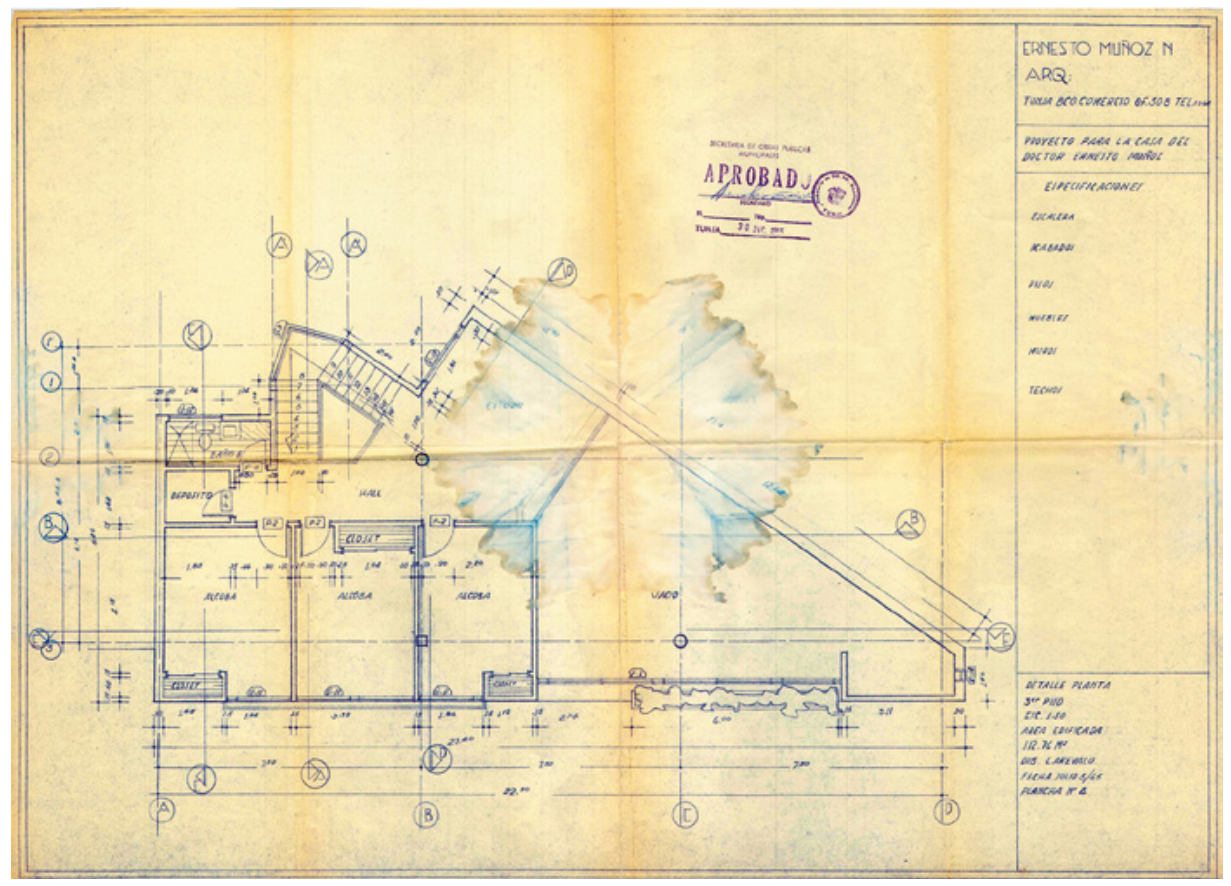

Figura 2. Plano original de la casa de Ernesto Muñoz Navarro. Fuente: Marcela Pinilla. 


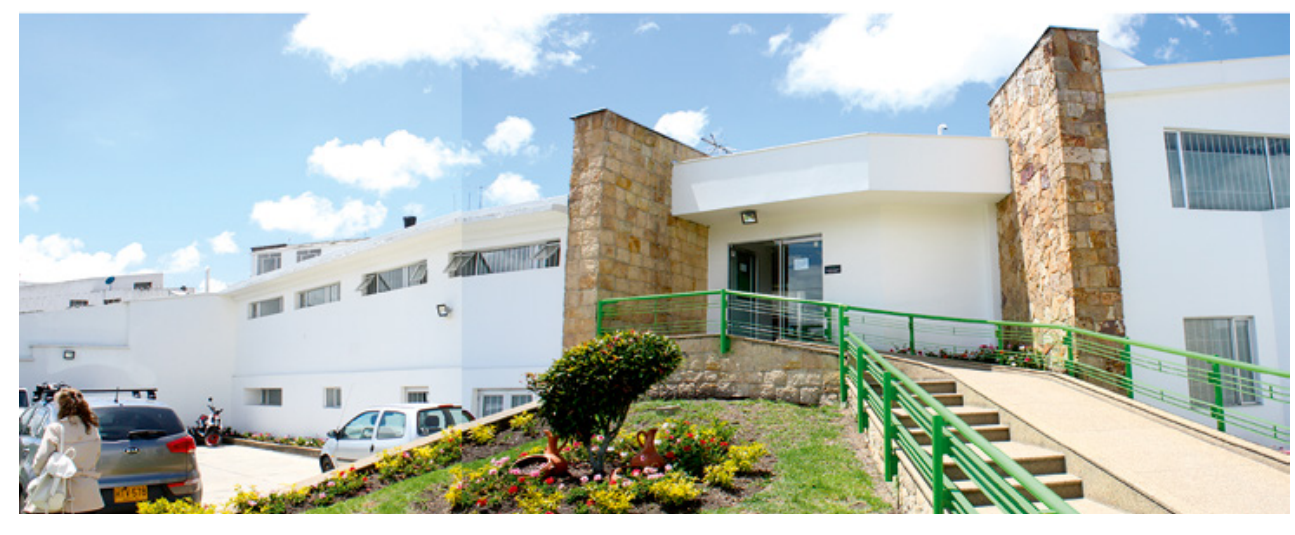

Figura 3. Casa destinada por varios años a los gerentes de Gaseosas Postobón. Fuente: Marcela Pinilla.

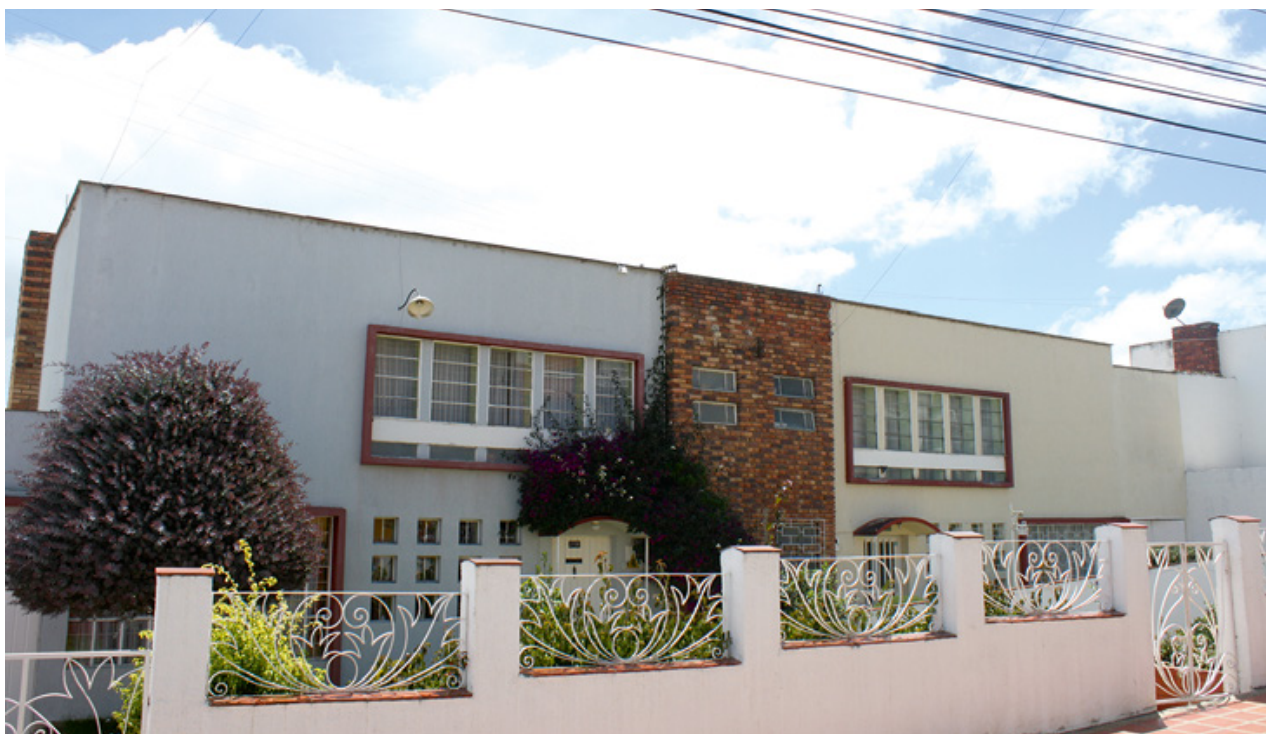

Figura 4. Casas en serie construidas por el Ingeniero Arquitecto Ernesto Muñoz Navarro.

Fuente: Marcela Pinilla. 


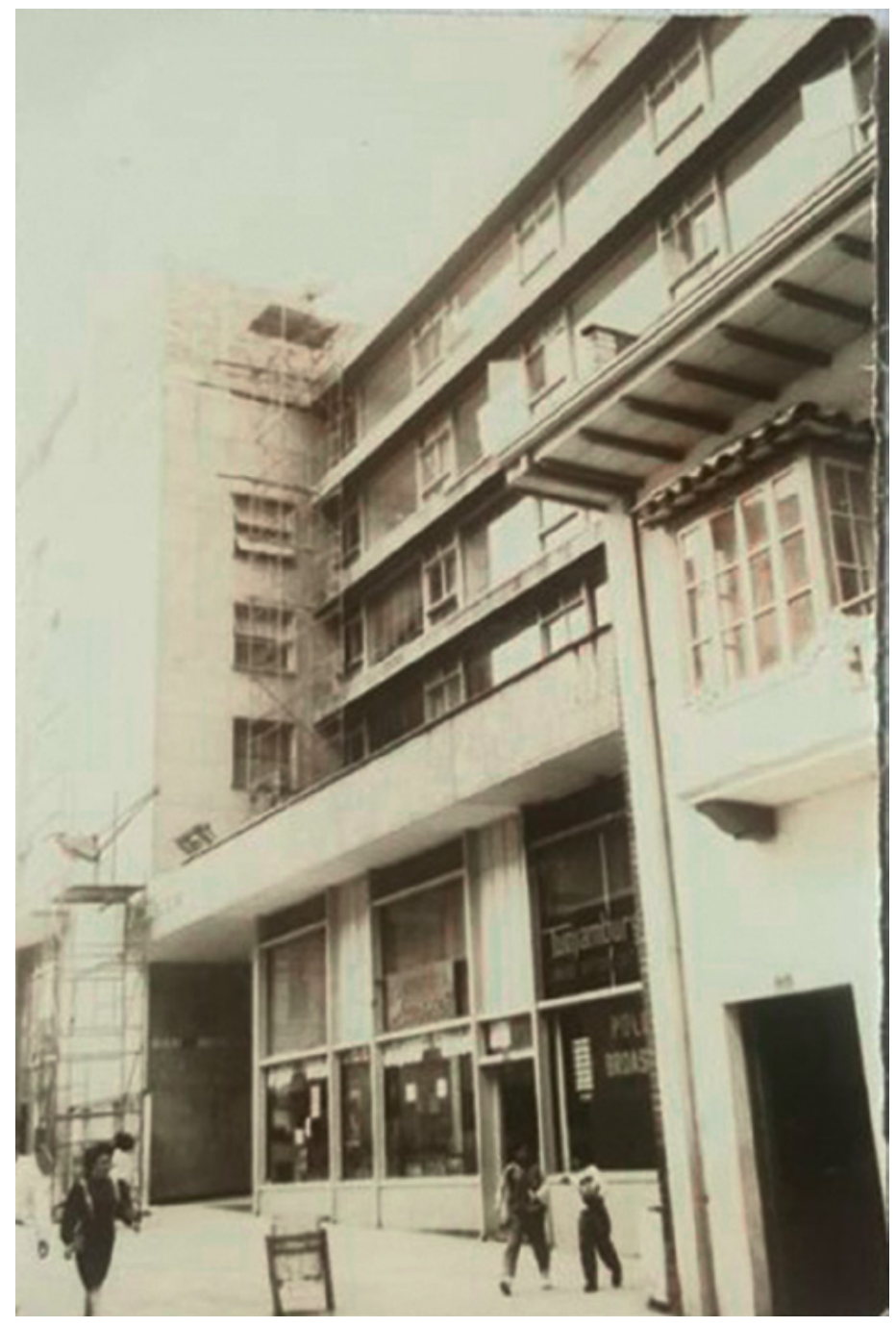

Figura 5. Banco del Comercio, 1991 (FER) Fuente: Marcela Pinilla. 


\section{CONCLUSIÓN}

La falta de reconocimiento de la arquitectura moderna como algo propio, como parte de la identidad tunjana, aunque no llegue a la categoría de Bien de Interés Cultural, representa un problema para la identidad arquitectónica de la ciudad. Desconocer la arquitectura moderna en el desarrollo de las ciudades significa la pérdida de la memoria histórica, pues se está ignorando su valor testimonial y estético. Por otra parte, debe reconocerse que la arquitectura moderna en Colombia en su momento más influyente cambió la vida de la ciudadanía y la imagen de las ciudades a lo largo del siglo XX.

Es una arquitectura que se desarrolló a partir de influencias extranjeras y enseñanzas de diferentes partes del mundo, que consolidaron un estilo propio de desarrollo arquitectónico. En este sentido, desconocer el modernismo en nuestras ciudades, sería ignorar el mérito de los arquitectos colombianos que crearon un estilo propio y particular, que hoy en día hace parte de nuestra identidad urbanística. En conclusión, si la arquitectura moderna continúa demoliéndose, se borrará irremediablemente las configuraciones infraestructurales que surgieron a raíz del desarrollo histórico de nuestro país.

Para el caso de Tunja, es de resaltar que el Plan Especial de Manejo y Protección (PEMP) en el año 2012, haya reconocido el valor de singularidad de algunas edificaciones de arquitectura moderna, que incluyen algunas de las obras del Arquitecto e Ingeniero Ernesto Muñoz Navarro, reseñadas en el presente documento, como el actual edificio de la Secretaría de Educación de Boyacá; y, además, que desde el PEMP, se les haya asignado el nivel 1 de intervención, lo que, en teoría, protege las edificaciones de la demolición y de intervenciones inadecuadas que perjudiquen la idea original de diseño. No obstante, no garantiza la apropiación de esta arquitectura por parte de la comunidad tunjana, que es lo que realmente permite la conservación de este patrimonio a largo plazo.

De ahí la importancia de esta investigación y de la amplia difusión de sus resultados a la ciudadanía tunjana, pues permitirá sacar a la luz la obra de arquitectos modernos que hicieron un importante aporte al desarrollo del espectro urbanístico de la bella ciudad de Tunja. 


\section{REFERENCIAS BIBLIOGRÁFICAS}

Arango, S. (1997). Arquitectura colombiana de los años 30 y 40: la modernidad como ruptura. Credencial historia $N^{\circ} 86$.

Caballero, J., \& Fonseca, L. (2001). Aproximación operativa al invetario - valoración del patrimonio construido. PROA.

García de Moncada, D. (2007). La Arquitectura y el poder en Bogotá durante el Gobierno del General Gustavo Rojas Pinilla, 1953- 1957. (U. Católica, Ed.) Revista de Arquitectura. Obtenido de http:// www.redalyc.org/pdf/1251/125112650004.pdf

García de Moncada, D. (2007). La arquitectura y el poder en Bogotá durante el gobierno del general Gustavo Rojas Pinilla, 1953-1957. Revista de arquitectura, Universidad Católica, 9.

Henao, E., \& Llanos, I. (2008). Sentido y vigencia de la arquitectura moderna colombiana. (M. d. cultura, Ed.) Ciudad y arquitectura moderna en Colombia 1950-1970. Presencia y vigencia del patrimonio moderno.

Hernández, A. (2019). La arquitectura del Movimiento Moderno: entre la desaparición y la reconstrucción. Apuntes, 156-179.

Hidalgo, A. (2014). Tunja: transformación urbana a partir de la vivienda obrera (1940-1957). Tunja : Universidad de Boyacá.

Hidalgo, A. (2015). Tres elementos modernos del patrimonio urbano difuso en Tunja. Territorios $N^{\circ} 33$. Recuperado el 14 de marzo de 2019, de https://revistas.urosario.edu.co/xml/357/35741605002/ index.html . 
Hidalgo, p. (2014). Tunja: transformación urbana a partir de la vivienda obrera (1940-1957).

López, M. C. (22 de octubre de 2009). DoCoMoMo Centro Oriente Colombia: Avance de la investigación sobre arquitectura moderna en Tunja (Boyacá). 2009. Tunja, Boyaca.

Medina Roa, J. (2016). Tunja desde 1900. Tunja: Consejo editorial de autores boyacenses CEAB.

Mendoza Laverde, C. (2008). La pérdida de la tradición moderna en la arquitectura colombiana: Bogotá y alrededores, y departamentos de Boyacá, Cundinamarca, Huila y Tolima. Revista Apuntes, 21(2).

Mendoza Laverde, C. (2008). Se destruye el legado del movimiento moderno en Colombia ¿Se conserva por decreto o por sus valores?

Quevedo Forero, E. (1999). Rojas Pinilla en Boyacá. Crónicas analistas. Tunja: Jotamar Ltda.

Redacción El Tiempo. (10 de marzo de 2000). Rojas Pinilla y Boyacá. El Tiempo, págs. www.eltiempo. com/archivo/documento/MAM-1280540.

Saldarriaga Roa, A. (1999). Arquitectura colombiana en el siglo XX: edificaciones en busca de ciudad. Revista Credencial Historia.

Saldarriaga Roa, A. (2017). La arquitectura en Colombia en varios tiempos. Credencial historia No 334.

Samper Martínez, E., \& Ramírez Nieto, J. (2000). Arquitectura moderna en Colombia: época de oro. Diego Samper Ediciones. 